\title{
Research Paper: Comparing Three Different Methods of Dressing for Skin Graft Donor Site
}

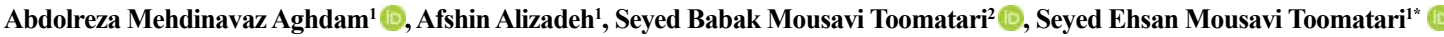

1. Department of General Surgery, Imam Reza Hospital, Tabriz University of Medical Sciences, Tabriz, Iran.

2. Department of General Surgery, Faculty of Medicine, Zanjan University of Medical Sciences, Zanjan, Iran.

\begin{tabular}{|l|l|}
$\begin{array}{l}\text { use yourdevice to scan } \\
\text { and read the article online }\end{array}$ & $\begin{array}{l}\text { Methods of Dressing for Skin Graft Donor Site. International Journal of Medical Toxicology and Forensic Medicine. 2020; } \\
\text { 10(2):29059. https://doi.org/10.32598/ijmtfm.v10i2.29059 }\end{array}$ \\
doi https://doi.org/10.32598/ijmtfm.v10i2.29059
\end{tabular}

(c) (1) (8)

Article info:

Received: 16 Feb 2020

First Revision: 25 Feb 2020

Accepted: 10 Mar 2020

Published: 28 Jun 2020

\section{Keywords:}

Skin graft donor site, Vaseline gauze, Nitrofurazone,

Mupirocin 2\%

\section{ABSTRACT}

Background: There are various methods to dress the Skin Graft Donor Site (SGDS), but some are very expensive and not available in Iran. This study aimed to compare three different dressings for the management of the SGDS.

Methods: Ninety-six patients participated in the study, and they were randomly divided into three groups. Donor sites were dressed with one of these methods: Vaseline gauze or method A, mupirocin $2 \%$ or method $\mathrm{B}$, and nitrofurazone or method $\mathrm{C}$. In each method, the dressing layer was covered by 5 layers of dry gauze. The three groups were compared regarding the epithelialization time, infection, pain, and cost.

Results: The epithelialization time was significantly longer in group A compared with groups $\mathrm{B}$ and $\mathrm{C}(\mathrm{P}<0.05)$, but there was no significant difference between groups $\mathrm{B}$ and $\mathrm{C}(\mathrm{P}<0.05)$. There was no significant difference between the three groups in infection and pain at rest, activity, and dressing time $(\mathrm{P}<0.05)$. There was a substantial difference between the three groups in the cost of dressing as method A was cheaper.

Conclusion: Due to the lower cost of Vaseline gauze and no significant difference in infection, dressing adhesion to the skin, and amount of pain between three dressing methods, Vaseline gauze is a preferred method to skin graft donor site dressing.

\section{Introduction}

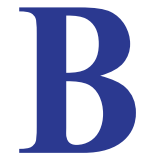

urns and related injuries are still one of the most important health problems due to their high rate of complications, prognosis, large number, long treatment duration, and the functional, psychological, and social effects $[1,2]$
Split-Thickness Skin Graft (STSG) has made a significant revolution in burn care [3] and accepted as a reconstructive procedure with a lot of benefits, such as less healing time and scar [4]. So it is widely used for wound cover [5].

Skin Graft Donor Site (SGDS) is an annoying problem for patients, and they usually complain about the donor site due to the risk of infection, pain, and poor cosmetic outcomes [6]. Partial-thickness wounds are created in

* Corresponding Author:

Seyed Ehsan Mousavi Toomatari, MD.

Address: Department of General Surgery, Imam Reza Hospital, Tabriz University of Medical Sciences, Tabriz, Iran.

Tel: +98 (914) 3882358

E-mail: semoosavit@gmail.com 
SGDS that must be treated by re-epithelialization. The epithelialization takes $10-14$ days to complete $[7,8]$. Various dressings have been suggested for managing these wounds [9].

We designed this study due to the lack of new drugs, their high costs, and the lack of specific treatment protocol in Iran. Our study aimed to compare three different dressings of Vaseline gauze, mupirocin 2\% ointment soaked mesh gauze, and nitrofurazone soaked mesh gauze. We intended to determine the best method of dressing the donor site considering the epithelialization time, pain, infection, and cost.

\section{Materials and Methods}

Individuals with inclusion criteria were recruited by a non-random sample method. Then, the random allocation was done to form study groups. In this study, a block randomization method (including quadruple blocks) was used.

Between March 2018 and March 2019, 96 patients with the second- and third-degree burns lesser than $20 \%$ TBSA (total body surface area) who were operated in any kind of reconstructive operations with STSG donor sites were included in this clinical trial study. Patients with diabetes mellitus, aged over 60 or under 16 years, under active immunosuppressive therapy, unable to estimate the amount of the pain, pregnant, burns greater than $20 \%$, and the first- and fourth-degree burns degree were excluded from the study.

The patients were divided into three equal groups. A manual dermatome was used to harvest an STSG from the thigh in a standard manner at $0.36-0.43 \mathrm{~mm}$ thickness, and the donor sites were dressed with one of the following methods intraoperatively:

Group A, Vaseline gauze, which was covered by 5 layers of gauze,

Group B, mupirocin 2\% ointment soaked mesh gauze, which was covered by 5 layers of dry gauze,

Group C, nitrofurazone, soaked mesh gauze, which was covered with 5 layers of dry gauze.

Patients were followed after operation for three days, and then the dressing was changed once daily to check the wound until epithelization. The same surgeon performed the donor site dressing. Wound epithelization was visually evaluated by the study researcher, who was unaware of the dressing group.
The skin donor site was assessed for the dressing time, infection, pain, dressing cost, dressing adhesion to the donor site, and hospitalization duration (until healing donor site). Visual Analog Scale (VAS) score was used to assess pain at rest, dressing, and activity.

If the clinical symptoms of erythema, exudate, fever, or increased pain were spotted, the patients would be evaluated for infection by wound swab and culture.

\section{Statistical analysis}

All obtained data were analyzed in SPSS V. 23. The results are expressed as Mean $\pm \mathrm{SD}$ or number and percentage. The Kolmogorov-Smirnov test was used to assess the normal distribution of data. The Chi-square test, Fischer exact test, and ANOVA test were used to compare data between groups. P values of less than 0.05 were considered statistically significant.

\section{Results}

Ninety-six patients were enrolled in the study, 32 in each group. Table 1 lists the demographic data of study groups. There were no significant differences between the three groups concerning the sex, age, degree of burning, and average \%TBSA.

Clinical symptoms of infection were discharge (group A: 1; group B: 0; group C: 1), erythema (group A: 2; group B: 1 ; group C: 1 ), and fever (group A: 1; group B \& C: 0$)$. Infection of the skin graft donor site occurred in 4 patients (4.2\%); there were two infections in group $\mathrm{A}$, one infection in group $\mathrm{B}$, and one infection in group C. The difference between groups was not statistically significant $(\mathrm{P}=0.79)$.

Dressing adhesion to skin graft donor site, VAS score, and duration of dressing are presented in Table 2. The mean duration of hospitalization in group $\mathrm{A}$ was $17.96 \pm 1.4$ days (range: $15-21$ days), group $B, 16.43 \pm 1.86$ days (range: 13-20), and group C, $16.9 \pm 1.55$ days (range: 14-20). This figure was significantly higher in group $A$ compared with group $B$ and $C(P<0.05)$, but there was no significant difference between groups $\mathrm{B}$ and $\mathrm{C}(\mathrm{P}=0.6)$.

Tables 3 and 4 present the epithelialization time and dressing cost. The duration of epithelialization in the Vaseline group was significantly higher than mupirocin $2 \%$ and nitrofurazone groups. Still, there was no significant difference between mupirocin $2 \%$ and nitrofurazone groups in this regard (Table 3). Dressing cost in mupirocin $2 \%$ group was significantly higher than the 
Table 1. Demographic data of study groups

\begin{tabular}{|c|c|c|c|c|c|}
\hline \multicolumn{2}{|c|}{ Variable } & Group A (Vaseline) & Group B (Mupirocin 2\%) & $\begin{array}{c}\text { Group C (Nitrofura- } \\
\text { zone) }\end{array}$ & $\mathbf{P}$ \\
\hline \multicolumn{2}{|c|}{ Age (y) } & $29.31 \pm 9.52$ & $34.81 \pm 10.27$ & $30.37 \pm 10.02$ & 0.069 \\
\hline \multirow{3}{*}{ Sex } & Male & $17(53.13)$ & $18(56.25)$ & $20(62.5)$ & \\
\hline & & & & & 0.74 \\
\hline & Female & $15(46.87)$ & $14(43.75)$ & $12(37.5)$ & \\
\hline \multirow{3}{*}{$\begin{array}{l}\text { Degree of } \\
\text { burning }\end{array}$} & II & $12(37.5)$ & $14(43.75)$ & $13(40.62)$ & \\
\hline & III & $11(34.3)$ & $12(37.5)$ & $10(33.33)$ & 0.89 \\
\hline & $\|\&\|$ & $9(28.13)$ & $6(18.75)$ & $9(28.13)$ & \\
\hline \multicolumn{2}{|c|}{ Average \%TBSA } & $11.21 \pm 4.65(4-20)$ & $11.59 \pm 4.54(5-20)$ & $11.31 \pm 4.48(5-20)$ & 0.07 \\
\hline
\end{tabular}

Table 2. Dressing adhesion to skin graft donor site, VAS score, and duration of dressing

\begin{tabular}{|c|c|c|c|c|c|}
\hline \multicolumn{2}{|c|}{ Variable } & \multirow{2}{*}{$\begin{array}{c}\text { Group A } \\
2.1 \pm 0.76\end{array}$} & \multirow{2}{*}{$\begin{array}{l}\text { Group B } \\
2.06 \pm 0.75\end{array}$} & \multirow{2}{*}{$\begin{array}{c}\text { Group C } \\
2.5 \pm 0.8\end{array}$} & \multirow{2}{*}{$\begin{array}{c}\mathbf{P} \\
0.62\end{array}$} \\
\hline & Rest time & & & & \\
\hline Visual analog scale & Dressing time & $3.71 \pm 0.85$ & $3.59 \pm 0.75$ & $3.71 \pm 0.88$ & 0.78 \\
\hline & Activity time & $2.87 \pm 0.79$ & $2.96 \pm 0.78$ & $2.93 \pm 0.8$ & 0.89 \\
\hline \multicolumn{2}{|c|}{ Duration of dressing } & $15.15 \pm 3.51$ & $15.28 \pm 4.02$ & $14.93 \pm 3.02$ & 0.92 \\
\hline \multirow{3}{*}{ Dressing adhesion } & Low & $12(37.5)$ & 15 (46.9) & $16(50)$ & \multirow{3}{*}{0.06} \\
\hline & Medium & $9(28.1)$ & $14(43.8)$ & $13(40.6)$ & \\
\hline & High & $11(34.4)$ & $3(9.4)$ & $3(9.4)$ & \\
\hline
\end{tabular}

Table 3. The duration of epithelialization

\begin{tabular}{cccc}
\hline & \multicolumn{2}{c}{ One-way Analysis of Variance } \\
\cline { 2 - 3 } Dressing & Epithelialization Time & F & P \\
\hline Vaseline & $14.03 \pm 1.12$ & & 0.001 \\
Mupirocin 2\% & $12.53 \pm 1.24$ & 13.12 & \\
Nitrofurazone & $12.77 \pm 1.44$ & & \\
\hline
\end{tabular}

\begin{tabular}{cccc}
\hline & \multicolumn{3}{c}{ Scheffe } \\
Dressing & Dressing & Mean Difference & P \\
\cline { 2 - 4 } & Mupirocin 2\% & 1.5 & 0.001 \\
Vaseline & Nitrofurazone & 1.31 & 0.001 \\
Mupirocin 2\% & Nitrofurazone & -0.18 & 0.84 \\
\hline
\end{tabular}

Data are presented as Mean \pm SD. 
Table 4. The dressing cost

\begin{tabular}{|c|c|c|c|}
\hline \multicolumn{4}{|c|}{ One-way Analysis of Variance } \\
\hline Dressing & Dressing Cost, Rial (US Dollar) & $\mathbf{F}$ & $\mathbf{P}$ \\
\hline Vaseline & $133400 \pm 5660(1.2 \$)$ & & \\
\hline Mupirocin 2\% & $505000 \pm 188200$ (4.16\$) & 57.02 & 0.001 \\
\hline Nitrofurazone & $329000 \pm 139600(2.75 \$)$ & & \\
\hline
\end{tabular}

\begin{tabular}{|c|c|c|c|}
\hline \multicolumn{4}{|c|}{ Scheffe } \\
\hline Dressing & Dressing & Mean Difference & $\mathbf{P}$ \\
\hline Vaseline & $\begin{array}{l}\text { Mupirocin 2\% } \\
\text { Nitrofurazone }\end{array}$ & $\begin{array}{l}-37.15 \\
-19.56\end{array}$ & $\begin{array}{l}0.001 \\
0.001\end{array}$ \\
\hline Mupirocin 2\% & Nitrofurazone & 17.59 & 0.001 \\
\hline
\end{tabular}

Data are presented as Mean \pm SD

Vaseline and nitrofurazone groups, also dressing cost in the nitrofurazone group was considerably higher than the Vaseline group (Table 4).

\section{Discussion}

STSG is one of the most common techniques for the replacement of skin damaged following burns [10, 11] because it has many benefits such as fast healing, acceptable scar, and low complications [11-13]. A partialthickness wound is created in the skin graft donor site that is healed by re-epithelialization. It takes 10-14 days to complete epithelialization $[14,15]$.

Dressing is an acceptable method to protect the wound and reduce the healing duration, pain, infection, and cost $[2,16]$. Various studies reported that several dressings are appropriate for this aim, including classical gauzes, modern silicone dressing, alginates, and hydrofibers [17].

The findings of the current study showed that epithelialization was similar in the mupirocin $2 \%$ and nitrofurazone dressing. Still, in both dressings, epithelialization time was significantly shorter than the Vaseline gauze dressing.

Roberto Cuomo et al. found that epithelialization and recovery time in fibrillary tabotamp was better and faster than Vaseline gauze [18]. Hassanpour et al. reported similar results [4]. Also, Masella et al. showed that hydrocolloid dressing had the greatest re-epithelialization outcome [11]. Brolmann et al. demonstrated that hydrocolloid dressing is preferable to alginate, film, gauze, hydrofibers, and silicone dressing due to better and faster re-epithelialization [17].
Our result showed that the amount of pain was not significantly different between the three groups in dressing, resting, and activity time. However, the pain in the Vaseline dressing was slightly higher than mupirocin. Unlike our findings, Hassanpour et al. reported that pain severity was significantly lower in nitrofurazone dressing compared to Vaseline gas [4]. Beiraghi-Toosi et al. showed dressing with Vaseline gauze was less painful than fine mesh gauze [19]. According to Uysal et al. results, pain severity was the least in nitrofurazone dressing [20].

Our study demonstrated that the incidence of infection and dressing adhesion to skin with Vaseline was higher than mupirocin and nitrofurazone, but this difference was not statistically significant. Unlike our findings, Hassanpour et al. showed that dressing with nitrofurazone had the least secretion and infection [4]. Brolmann et al. found that the infection rate with gauze dressing was higher than other dressings such as hydrocolloid, hydrofibers, or silicone [17]. According to Roberto Cuomo et al. , the rate of infection in the fibrillary tabotamp dressing was lower than the Vaseline dressing [18].

The finding of the current study showed that the cost of Vaseline gauze dressing was significantly less than the other two methods. Different studies have demonstrated similar results $[18,19]$. For example, Beiraghi-Toosi et al. reported the cost of dressing with Vaseline is low [19]. About the costs, Hassanpour et al. found no statistically significant difference between nitrofurazone and Vaseline gauze dressing [4]. 


\section{Conclusion}

Although epithelialization time and duration of hospitalization with mupirocin $2 \%$ and nitrofurazone are lower than Vaseline gauze, due to lower cost of vaseline gauze and no significant difference in infection, dressing adhesion to the skin, and amount of pain between three dressing methods, Vaseline gauze may be preferred as an alternative method to skin graft donor site dressing.

\section{Ethical Considerations}

\section{Compliance with ethical guidelines}

The Ethics Committee of Tabriz University of Medical Sciences (IR.TBZMED.REC.1398.278) and Iranian Registry of Clinical Trials (IRCT20190325043107N5) approved this study.

\section{Funding}

This research did not receive any specific grant from funding agencies in the public, commercial, or not-forprofit sectors.

\section{Author's contributions}

Conceptualization: Abdolreza Mehdinavaz Aghdam, Afshin Alizadeh Milani; Methodology: Abdolreza Mehdinavaz Aghdam, Afshin Alizadeh Milani, Seyed Ehsan Mousavi Toomatari; Writing - review \& editing: Abdolreza Mehdinavaz Aghdam, Seyed Babak Mousavi Toomatari, Seyed Ehsan Mousavi Toomatari; Supervision: Abdolreza Mehdinavaz Aghdam, Seyed Ehsan Mousavi Toomatari; Data collection: Afshin Alizadeh Milani; Data analysis: Seyed Ehsan Mousavi Toomatari.

\section{Conflict of interest}

The authors declared no conflict of interest.

\section{Reference}

[1] Ungureanu M. Concepts in local treatment of extensive pediatrics burns. J Med Life. 2014; 7(2):183-91. [PMID] [PMCID]

[2] Kazanavičius M, Cepas A, Kolaityte V, Simoliuniene R, Rimdeika $R$. The use of modern dressings in managing split-thickness skin graft donor sites: A single-centre randomised controlled trial. J Wound Care. 2017; 26(6):281-91. [DOI:10.12968/ jowc.2017.26.6.281] [PMID]
[3] Schneider JC, Qu HD. Neurologic and musculoskeletal complications of burn injuries. Phys Med Rehabil Clin N Am. 2011; 22(2):261-75. [DOI:10.1016/j.pmr.2011.01.003] [PMID]

[4] Hassanpour SE, Moosavizadeh SM, Yavari M, Hallaj Mofrad HR, Fadaei AR. Comparison of three different methods of dressing for partial thickness skin graft donor site. World J Plast Surg. 2013; 2(1):26-32.[PMID] [PMCID]

[5] McBride CA, Kimble RM, Stockton K. Three donor site dressings in pediatric split-thickness skin grafts: Study protocol for a randomised controlled trial. Trials. 2015; 16:43 [DOI:10.1186/s13063-015-0557-9] [PMID] [PMCID]

[6] Barrit DP, Birke-Sorensen H. Dressings for split thickness skin graft donor sites: A comparison of three options. World J Plast Surg. 2014; 14(2):15-20. https://pdfs.semanticscholar. org/4262/c52e3192f59a19d7328a6a97c7a8e6b939ab.pdf

[7] Palmu R, Partonen T, Suominen K, Saarni SI, Vuola J, Isometsa E. Health-related quality of life 6 month after burns among hospital;ized patients: Predicitve importance of mental disorders and burn severity. Burns. 2015; 41(4):742-8. [DOI:10.1016/j.burns.2014.11.006] [PMID]

[8] Caliot J, Bodin F, Chiriac S, Correia N, Poli-Mérol ML, François-Fiquet C. Split-thickness skin graft donor site: Which dressing use? Ann Chir Plast Esthet. 2015; 60(2):140-7. [DOI:10.1016/j.anplas.2014.09.006] [PMID]

[9] Hashemi F, Dolatabad FR, Yektatalab S, Ayaz M, Zare N, Mansouri P. Effect of orem self-care program on the life quality of burn patients referred to ghotb-al-din-e-shirazi burn center, Shiraz, Iran: A randomized controlled trial. Int J Community Based Nurs Midwifery. 2014; 2(1):40-50. [PMID] [PMCID]

[10] Demirtas Y, Yagmur C, Soylemez F, Ozturk N, Demir A Management of split-thickness skin graft donor site: A prospective clinical trial for comparison of five different dressing materials. Burns. 2010; 36(7):999-1005. [DOI:10.1016/j. burns.2009.05.017] [PMID]

[11] Masella PC, Balent EM, Carlson TL, Lee KW, Pierce LM Evaluation of six split-thickness skin graft donor site dressing materials in a swine model. Plast Reconstr Surg Glob Open. 2014; 1(9):e84. DOI:10.1097/GOX.0000000000000031] [PMID] [PMCID]

[12] Dornseifer U, Lonic D, Gerstung TI, Herter F, Max Fichter A, Holm C, et al. The ideal split-thickness skin graft donorsite dressing: A clinical comparative trial of a modified polyurethane dressing and aquacel. Plast Reconstr Surg. 2011 128(4):918-24. [DOI:10.1097/PRS.0b013e3182268c02] [PMID]

[13] De Carvalho VF, Paggiaro AO, Isaac C, Gringlas J, Ferreira MC. Clinical trial comparing 3 different wound dressings for the management of partial-thickness skin graft donor sites. J Wound Ostomy Continence Nurs. 2011; 38(6):643-7. [DOI:10.1097/WON.0b013e3182349d2f] [PMID]

[14] Starvrou D, Weissman O, Tessone A, Zilinsky I, Holloway S, Boyod J, et al. Health related quality of life in burn patiens-areview of the literature. Burns. 2014; 40(5):788-96. [DOI:10.1016/j.burns.2013.11.014] [PMID]

[15] Eskes AM, Brölmann FE, Gerbens LAA, Ubbink DT, Vermeulen $\mathrm{H}$. Which dressing do donor site wounds need? Study protocol for a randomized controlled trial. Trials. 2011 12: 229. [DOI: 10.1186/1745-6215-12-229] [PMID] [PMCID] 
[16] Uraloglu M, Livaoglu M, Agdogan O, Mungan S, Alhan E, Karacal N. An evaluation of five different dressing materials on split-thickness skin graft donor site and full-thickness cutaneous wounds: An experimental study. Int Wound J. 2014; 11(1):85-92. [DOI:10.1111/j.1742-481X.2012.01071.x] [PMID]

[17] Brolmann FE, Eskes AM, Goslings JC, Niessen FB, de Bree $\mathrm{R}$, Vahl AC, et al. Randomized clinical trial of donor-site wound dressings after split-skin grafting. Br J Surg. 2013; 100(5):619-27. [DOI:10.1002/bjs.9045] [PMID]

[18] Cuomo R, Grimaldi L, Cesare B, Nisi G, D’Aniello C. Skin graft donor site: A procedure for a faster healing. Acta Biomed. 2017; 88(3):310-14. [DOI:10.23750/abm.v88i3.5736] [PMID] [PMCID]

[19] Beiraghi-Toosi A, Motamedolshariati M, Einbeigi N. Dressing of the donor site of partial-thickness skin grafts with Vaseline gauze and fine mesh gauze. Med J Mashhad Uni Med Sci. 2016; 59(5):322-7. http://eprints.mums.ac.ir/1449/

[20] Uysal AC, Alagoz MS, Orbay H, Sensoz O. An alternative dressing material for the split-thickness skin graft donor site: Oxidized regenerated cellulose. Ann Plast Surg. 2006; 57(1):60-4. [DOI:10.1097/01.sap.0000208945.12083.59] [PMID] 